\title{
JERARQUIAS DE COLOR Y MESTIZAJE en la amazonia occidental colombiana
}

\author{
Margarita Chaves Chamorro \\ INVESTIGADORA ICANH \\ mchaves@mincultura.gov.co
}

\begin{abstract}
Resumen
[ N EL CONTEXTO AMAZÓNICO Y EN EL LATINOAMERICANO, RAZA Y ETNICIDAD SON CATEGORÍAS [ que presentan limitaciones conceptuales para aproximarnos al orden étnico y racial creado por el mestizaje, un orden caracterizado por la fluidez y la ambigüedad en el que cualquier reclamo de adscripción dentro de las categorías indio, negro, mestizo o blanco está lejos de ser evidente. Este artículo intenta mostrar las ventajas que representa la incorporación de la categoría de color, como una categoría específica, complementaria a aquellas de raza, etnicidad y clase. El color es importante para el análisis de la dinámica entre grupos diferentes cuyas fronteras son fluidas, y entre individuos (auto) percibidos como indio, negro, mestizo y blanco, pero en particular en contextos en los que los rasgos fenotípicos asociados con estereotipos raciales, etnicidad y clase no son suficientes para establecer jerarquías mestizas o entre mestizos.
\end{abstract}

PALABRAS CLAVE: color, mestizaje, identidad, etnicidad, indígenas, colonos, amazonia colombiana.

\section{Abstract}

N THE AMAZONIA, AS WELL AS IN the LATIN AMERICAN CONTEXT, RACE AND ETHNiCITY ARE conceptually limited categories for dealing with ethnic and racial orders created by mestizaje; an order characterized by fluidity and ambiguity in which any claims at inclusion within the categories of Indian, black, mestizo or white is far from being evident. The article tries to show the advantages presented by incorporating the category of 'color', as a specific category complementing those of race, ethnicity and class. Color is important for analyzing the dynamics between different groups with fluid frontiers and between individuals who perceive themselves as Indian, black, mestizo and white, but more specifically in contexts where phenotypic traits associated to racial stereotypes, ethnicity or class are not sufficient to establish hierarchies within or between mestizos.

KEY wORDS: color, miscegenation, mestizaje, identity, ethnicity, indigenous peoples, homesteaders, Colombian Amazonia.

\section{Revista Colombiana de Antropología}

Volumen 38, enero-diciembre 2002, pp. 189-216 


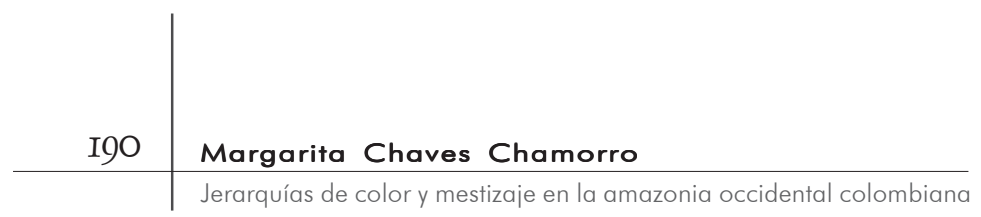

En una sociedad en la cual privilegio y poder son monopolio de los de piel blanca, Esperanza es muy consciente de su piel oscura, y de su indianidad. No obstante, su indianidad no está basada en una identidad étnica, sino en distinciones de raza/clase que se han desarrollado desde la conquista española de México y que continúan afirmando el poder del privilegio de la piel blanca.

RUTH BEHAR, Translated woman

\section{INTRODUCCIÓN*}

N

TRABAJO DE INVESTIGACIÓN PRETENDE DESARROLLAR ELEMENTOS PARA una antropología de la identidad y la representación en la amazonia occidental colombiana, en particular de los indígenas y los colonos de las áreas de Putumayo y Guaviare. Existe la intención de incluir luego en el análisis las dinámicas sociales de sectores urbanos, pues ellas cumplen un papel determinante en las representaciones e identidades que construyen los grupos de nuestro interés.

Para los propósitos de este artículo, la Amazonia occidental colombiana es la región cercana al piedemonte de la cordillera que hace parte de los departamentos de Caquetá, Putumayo y Guaviare, la cual ha estado sujeta a procesos de colonización extractiva y campesina y a una intensa dinámica de poblamiento y mestizaje físico y cultural entre indígenas y colonos, tanto en las áreas rurales como alrededor de centros urbanos numerosos y relativamente importantes. La tendencia hacia la concentración de la población en las capitales departamentales se corresponde con la de las instituciones del estado y el comercio en las mismas. Esta tendencia se ha acentuado en los últimos

\footnotetext{
* Este artículo hace parte de los resultados de investigación del proyecto Multiculturalidad y conflicto interétnico en la amazonia occidental colombiana, financiado por Colciencias y el ICANH. La primera etapa de investigación de este trabajo fue posible gracias al apoyo financiero de la Fundación Interamericana. Agradezco a las lectoras y los lectores de este manuscrito sus comentarios críticos y sugerencias, en particular a Marta Zambrano y Odile Hoffmann. Cualquier omisión e interpretación errada son responsabilidad mía.
} años debido a los procesos de desplazamiento que ocasiona el enfrentamiento armado entre la guerrilla, los paramilitares y el ejército en las áreas rurales. Allí, la actividad predominante es el cultivo y procesamiento de coca, organizada en pequeñas parcelas de producción doméstica que se articulan con fincas de mayor 


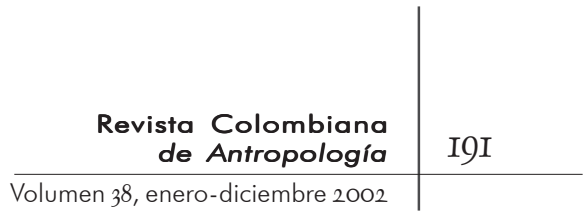

extensión controladas por patrones sobre quienes la guerrilla ejerce un amplio control. En la región, las cifras de población indígena son considerablemente reducidas comparadas con las de la población de colonos; sin embargo, los imaginarios que la construyen enfatizan sobre la importancia de lo indígena.

En general, hasta hace poco la aproximación al problema de la identidad de los indígenas de la región, y por defecto de los colonos, la ha enmarcado dentro de categorías de etnicidad y cultura, que subrayan la pertenencia de los indígenas a una de las diferentes etnias autóctonas del territorio, sustentada en su lugar de origen, continuidad histórica, cultura y lengua comunes. El resultado ha sido, con frecuencia, la esencialización de las identidades indígenas y la ausencia de análisis sobre las identidades que construyen los colonos, quienes al ser descritos como de origen mestizo se consideran no étnicos.

La discusión teórica sobre etnicidad e identidad dentro de lo que se ha denominado la posmodernidad -pero que de algún modo ya está presente en la propuesta de Fredrik Barth (I976) sobre la construcción de fronteras étnicas- ha mostrado que identidad-representación y las construcciones alrededor de la misma son siempre un discurso que articula -es resultado y momento a la vez-, relaciones de poder entre grupos sociales diferentes, hegemónicos y subalternos. Es decir, la identidad no sólo se construye en relación con la diferencia, sino en el contexto de relaciones de poder. En tal sentido, la identidad-representación, así como su esencialización y su crítica, son necesariamente políticas en la medida en que constituyen un momento central en la construcción de las relaciones de poder (este aspecto se discute en extenso en Hall, I990, I99I; para el caso particular de la amazonia colombiana véase Chaves, 1998, 200I).

No obstante, una discusión más profunda sobre la dinámica de las relaciones sociales en general e interétnicas en particular en el área de estudio, muestra que las perspectivas que ofrecen las categorías de etnicidad y cultura, a las que habría que añadir las de estatus y clase propias del análisis sociológico tradicional, no bastan para comprender de manera articulada -Marx diría orgánica- la realidad compleja de la interacción social en la amazonia occidental colombiana, tanto en una perspectiva histórica como en cuanto a los procesos más recientes. Ello en la medida en que el proceso de transculturación, resultado del encuentro entre colonizadores y colonizados en el proceso de ocupación 
del territorio por parte de los primeros, instituye un orden y una valoración de las posiciones de los sujetos que se sustenta en su construcción ideológica de alteridad que las categorías de etnicidad, cultura, estatus y clase no alcanzan a aprehender en toda su expresión.

El recuento y una doble reflexión sobre una experiencia personal de trabajo de campo en una comunidad indígena siona en el Putumayo pueden ayudar a ilustrar la cuestión.

En 1994, a mi llegada a Buenavista, un asentamiento indígena Siona a orillas del río Putumayo donde había realizado trabajo etnográfico años atrás, miembros de la familia donde usualmente me hospedo y viejos amigos llegaron a saludarme. Entre ellos se encontraba Jaime Erazo, un campesino colono, blanco, rubio y de ojos azules, venido de Samaniego, Nariño, y casado con una indígena del lugar. Portando un bastón de mando, similar al que usan los indígenas del Cauca, Jaime se presentó como el nuevo gobernador "indígena" del asentamiento. Este cargo, que para él representaba un honor, constituía el reconocimiento por parte de la comunidad de su adopción del modo de vida indígena tras i8 años de haber compartido con los siona sus espacios sociales y políticos (Chaves, I998: 282).

La primera reflexión sobre esta experiencia la enmarqué dentro del contexto de la dinámica social generada por los efectos de la promulgación de la constitución de I99I. Es decir, la identificación que Jaime Erazo hacía de sí mismo como indígena la interpreté como el intento de un colono por participar de los beneficios políticos y territoriales que dicha constitución había otorgado a los grupos indígenas. Así visto, el caso hacía evidente la tensión existente entre reconocimiento étnico y derechos políticos, presente ahora en los procesos de recreación y redefinición de identidad y representación entre los distintos actores de la región.

Sin embargo, el hecho particular de que Jaime Erazo se presentara y hubiera sido elegido como gobernador del resguardo siona de Buenavista era no sólo expresión de la intensidad de la interacción entre indígenas y colonos, sino que mostraba, además, que los procesos de recreación de identidad no podían ser aprehendidos únicamente desde una perspectiva instrumental, "como un mero reflejo de los procesos políticos", como lo señalé en ese entonces (Chaves, I998: 284). 


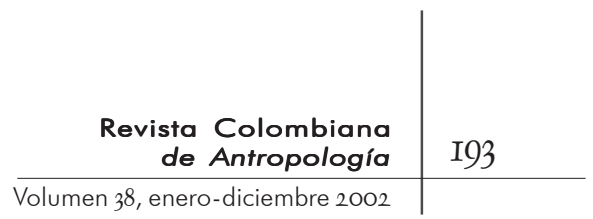

La segunda reflexión, en parte un análisis de ese primer texto, se refiere a la representación que hago de Jaime Erazo como un "colono blanco, rubio y de ojos claros". En esta descripción, a primera vista casi inocente, están implícitas, sin embargo, concepciones -en este caso de la antropóloga- sobre mestizaje y color que entran como características constitutivas de la identidad del colono, y que aquí me interesa explorar. ¿Habría sido tan sugerente el caso de Jaime Erazo si el color de su piel y sus rasgos hubieran sido como los de la gran mayoría de indígenas y colonos que se encuentran en el Putumayo? ¿Dónde hacer coincidir lo blanco de los rasgos de Erazo con su estatus de gobernador indígena? ¿Qué papel cumple el color -piel, ojos, cabelloen el contexto de procesos de re-indigenización a la orden del día en esa región? ¿Y cuál con los mestizos blancos cuando el referente étnico, que asocia fenotipo indígena y etnicidad, entra a ser valorado de manera positiva?

Dicho de otro modo, y de manera más general, en un contexto de intensa interacción interétnica una antropología de la representación del colono, o bien del mestizo, tanto en la práctica social y política de los distintos actores de la región como en el texto etnográfico de la antropóloga está lejos de ser evidente. La intención de este ensayo es, pues, dar algunas luces que hagan explícito lo implícito y, a la vez, permitan aprehender las categorías de mestizo y de color en sus determinaciones y manifestaciones múltiples.

El artículo intenta mostrar las ventajas que representa incorporar la categoría de color para aproximarnos a las identificaciones creadas dentro del mestizaje. Como categoría social y política, el color, al igual que la raza, es producto del régimen de verdad que construyó la colonización y expansión europea, en la que el color blanco, asociado al color de la piel del colonizador, se convierte en un marcador de la identificación del colonizado como no blanco -negro o de color- y de la subordinación social y política adherida al mismo.

En su uso cotidiano, el color es una categoría relacional difícil de aprehender, dado que el significado de su uso relativo -cuándo un individuo (moreno) aparece como más o menos blanco, o más o menos negro- depende de quién lo utiliza, para referirse a quién (a sí mismo o a otros), en qué contextos y con qué intenciones. Es decir, el color no construye un sistema único 
de significaciones sino sistemas diferentes, deslizándose del uno al otro de acuerdo con las posiciones de enunciación del mismo.

Ahora bien, aunque la referencia al tono más o menos claro de la piel o de los ojos es importante, el color no es sólo pigmentación y rasgos asociados con ella. De igual modo, aunque se relaciona con los rasgos físicos de una persona, lo que se encuentra detrás del mismo no es apenas apariencia racial, y probablemente no sea tampoco esta el elemento central que lo define. Ello porque el color hace parte de un sistema de contrastes significativos de poder, riqueza, estatus y otros valores, instituido por el colonialismo, el cual pervive en el poder del discurso instaurado históricamente, en el que lo blanco sirve como marca de un capital simbólico que confiere poder a quien lo reclama para hacer intercambios ventajosos, tanto materiales como simbólicos. Esta es la respuesta a la contradicción aparente que se evidencia cuando una misma persona se reclama como blanca y en otro momento como morena o negra, o para el caso, como india.

El color es importante para el análisis de la dinámica entre grupos diferentes cuyas fronteras son fluidas, y entre individuos (auto) percibidos como indio, negro, mestizo y blanco, en particular en contextos en los que los rasgos fenotípicos asociados con estereotipos raciales, etnicidad y clase no son suficientes para establecer jerarquías mestizas o entre mestizos. Mi propósito es esclarecer la complejidad de estos aspectos, al tiempo que surgen nuevos interrogantes.

El artículo está planteado en términos analíticos y deberá enriquecerse con datos históricos, sociológicos y etnográficos. Sin embargo, la literatura sobre las relaciones interétnicas y sobre su desarrollo histórico no es siempre bastante rica; esto último es particularmente cierto en lo que se refiere a las construcciones de las identidades étnicas o raciales de indios, blancos y colonos mestizos en la región de estudio. Mi análisis intenta proponer una salida con base en los datos actuales con que cuento, que hacen referencia, principalmente, a diferentes áreas del Putumayo. Sin embargo, con algunas variaciones considero que el esquema puede hacerse extensivo a Guaviare y Caquetá.

En la primera parte del artículo discuto brevemente el origen colonial de las categorías étnicas y raciales que han predominado en el contexto de los estados-nación latinoamericanos y sobre la pertinencia del color para aproximarnos a la estructura 


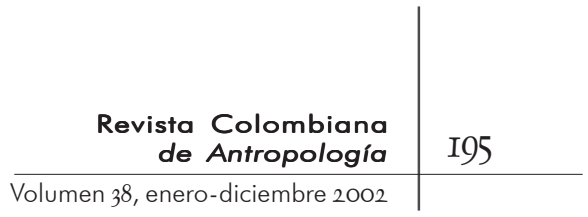

jerárquica de las mismas. La segunda utiliza este concepto en el análisis de la identidad de colonos e indígenas de la amazonia colombiana, en el intento de discernir las transformaciones que la reciente valoración positiva de lo indígena ha tenido en las construcciones de identidad y representación tanto de indígenas como de colonos de esta área. En particular, me pregunto por las jerarquías de mestizos, ya no en un contexto de blanqueamiento sino de indigenización, para lo cual la categoría de color resulta esclarecedora. Mis aproximaciones a este punto las presento más como hipótesis por ser confirmadas que como una interpretación validada. Mi argumento requerirá de modificaciones a medida que avance en el proceso de recolección de datos disponibles sobre el área de estudio, pero por lo menos espero mostrar que en esta área mestizaje y color están relacionados de manera sistemática el uno con el otro.

\section{Mestizaje y orden colonial EN LATINOAMÉRICA}

N I DISCUSIÓN SOBRE EL MESTIZAJE Y LA UTILIDAD DE LA CATEGOría de color para definir jerarquías dentro del mismo es, en gran parte, una extrapolación de análisis realizados alrededor de este aspecto por diferentes investigadores en relación con diversas situaciones y países del contexto latinoamericano (Lugo, I993; Lancaster, I992; Sasone 1997). En esos análisis se muestra la prevalencia de jerarquías de color dentro del mestizaje que ha construido a las sociedades latinoamericanas, al tiempo que se cuestiona la viabilidad misma del concepto de mestizaje para dar razón de esa construcción. De allí la importancia de considerar, aunque sea brevemente, el origen colonial de las categorías raciales de indio, negro, mestizo y blanco. Esto nos permite entender cómo el mestizaje y el color, producto de la consolidación de un orden colonial, da forma a la estructura actual de las sociedades latinoamericanas.

En la intensa mezcla racial y cultural que tuvo lugar antes y durante el periodo colonial, la estratificación social basada en el color desempeñó un papel importante en las sociedades coloniales latinoamericanas. Una vez apareció el mestizo como ser biológico y político-cultural, y el orden sociopolítico colonial 
se encontró plenamente establecido, indígenas y negros podían intentar convertirse o llegar a ser mestizos por medio del abandono de sus comunidades y la adopción de prácticas culturales y valores europeos, tales como la lengua española y el cristianismo, en un proceso que algunos autores han denominado blanqueamiento (Stutzman, I98I; Whitten, I985; Wade, I993). Durante el siglo dieciocho, el orden colonial clasificó a las personas mediante un intrincado sistema de graduación del color de la piel y de otros rasgos fenotípicos definidos en relación con los colonizadores europeos. En la así llamada sociedad de castas, el blanqueamiento y la limpieza de sangre asociada con las elites blancas -criollos americanos y españoles- eran claves en la posición social de los individuos, mientras que el oscurecimiento de la piel se relacionaba con la proximidad a los negros y a los indios y, por tanto, era signo de atraso, pobreza e ignorancia. Este orden jerárquico prevaleció hasta finales del siglo diecinueve, cuando entre las elites nacionales e imperiales se arraigaron concepciones específicamente raciales sobre las diferencias sociales y culturales.

A partir de las guerras de independencia, las elites criollas utilizaron el mestizaje -resultado genuino de la mezcla de razas que se dio en el nuevo mundo-como una ideología que promulgaba la igualdad y la libertad para redefinir como nacionales a las poblaciones de indios, negros y mestizos subalternos (Wade, I993; Klor de Alva, I995). Como discurso fue la narrativa de construcción nacional de los líderes criollos y mestizos de las nuevas naciones independientes, en su intento de oponer una identidad nacional mestiza al dominio imperial español. Pese a la inclusión predicada por el discurso del mestizaje, los indígenas y los negros fueron excluidos racial y culturalmente del proyecto nacional. Su bajo estatus y su falta de recursos configuraron su marginación de los centros de poder, por lo que muchos de ellos urgieron a sus descendientes mestizos y a algunos de sus miembros a abandonar sus comunidades y, cuando fue posible, a manipular estratégicamente sus identidades equívocas para alcanzar un estatus social más alto en un mundo dominado por la cultura y los valores occidentales. Este hecho, señalado por Klor de Alva (1995), revela el carácter construido-negociado de las identidades etno-raciales de los diferentes grupos de población que han estructurado y prevalecido en las sociedades latinoamericanas desde los tiempos coloniales. Hacia fines del siglo 


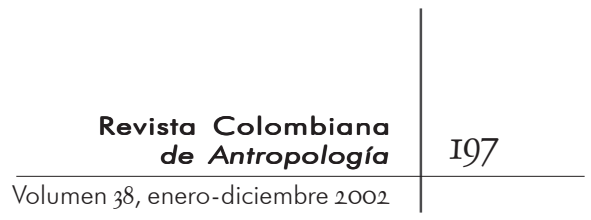

diecinueve se había establecido firmemente la estructura social que caracteriza hoy en día a la mayoría de los países latinoamericanos.

Whitten (1985) ha propuesto un modelo útil para entender la estructura etno-racial de las sociedades latinoamericanas contemporáneas, que sirve de referencia general. El orden social étnico/racial de las naciones puede representarse como una pirámide. En el tope de la jerarquía social se encuentra la oligarquía blanca que detenta un gran poder económico y político. Debajo está una clase alta de mestizos de piel clara, que sirve de barrera social selectiva para la capa más baja de la pirámide social/étnica. La mayor parte de la población de las naciones se compone de individuos mestizos, negros e indios, los dos últimos en la base de la pirámide. Mestizos, indios y negros buscan ascender socialmente por medio del blanqueamiento físico y cultural. El modelo de Whitten puede modificarse de acuerdo con variaciones nacionales y regionales, y usarse o como marco general de aproximación a las sociedades latinoamericanas o para comprender la dinámica de países particulares, tal como lo ha hecho Wade (1993) para el caso colombiano. La discusión de la tesis de este autor permite precisar la cuestión del color.

La ambigüedad es tal vez uno de los rasgos principales del orden racial creado por la mezcla racial. Dentro de una matriz mestiza la (auto) identificación es siempre fluida y las lealtades políticas de los sujetos inscritos en un orden tal son esencialmente inestables y desafían el intento de imposición de límites culturales rígidos entre grupos diferenciados. Como Wade bien lo anota para el caso de los indios y de los negros, las fronteras entre estos grupos y el resto de la sociedad siempre han estado en disputa y son ambiguas, a pesar del hecho de que cada uno de sus miembros tenga claros estereotipos de una típica persona negra o india, incluidos los mismos negros e indígenas. Este autor señala, con razón, la persistencia del color más allá del patrón de tolerancia y discriminación en contra de la gente negra. Sin embargo, sólo elabora dicha categoría con miras a entender el blanqueamiento físico y cultural como un medio de ascenso en la jerarquía social. Aquí, el color sólo es importante en relación con la discriminación como proceso social, en el cual los negros son clasificados e identificados como tales por una población mestiza y una elite blanca. Wade, y este es el punto particular sobre el que es necesario insistir, no desarrolla en su 
discusión de la noción de color la determinación de este último como una categoría mediante la cual los mismos negros establecen jerarquías mestizas/negras, si bien ilustra momentos en los que este es el caso (Wade, 1993: 319-322).

El color es una categoría fundamental en el contexto latinoamericano, dado que determina y media la reproducción de la doble correspondencia entre raza/etnicidad y clase/poder que ha dominado en las sociedades latinoamericanas desde los tiempos coloniales. Lancaster (I992) ilustra, para el caso de Nicaragua, cómo opera el color entre los mestizos y en la sociedad nicaragüense en general. Los nicas de la costa pacífica se consideran a sí mismos blancos -una minoría pequeña con poder-o mestizos, aun en casos en los que algunos de ellos podrían ser considerados como negros en otros contextos como el estadounidense. Al mismo tiempo, esos mismos nicas llaman negros a las minorías afrocaribeñas e indígenas que viven en la costa caribe. Etnicidad y raza son aquí suficientes para establecer la diferencia entre las dos áreas geográficas y para crear la verdad social de una jerarquía étnica y de color, en la cual el blanco y el mestizo de la costa pacífica están mejor situados en la estructura social y económica nicaragüense que los negros de la costa caribe. Según esto, la minoría blanca se encuentra en el tope de esta estructura, los mestizos en un nivel más bajo, y las minorías negras de la costa caribeña en la base, sin formar parte de la cultura mestiza nicaragüense, mayoritaria.

Sin embargo, en la construcción de una jerarquía mestiza el color cumple un papel central entre los nicaragüenses mestizos, igualmente situados en términos sociales y económicos y similares culturalmente. Términos de color tales como chele o rubio, blanco, moreno y negro se usan de manera diferente en distintas situaciones sociales. En todos ellos existe una valoración más alta del blanco y del color de piel claro, individualmente, dentro de la familia y más allá de la misma. Así, rasgos fenotípicos asociados con un estereotipo racial particular -por ejemplo, el color de los ojos, el cabello crespo- bien sea europeo, africano o indígena, se utilizan en contextos sociales para ubicar un individuo en la doble jerarquía social y mestiza. En particular, los estereotipos raciales o étnicos se tornan importantes en ciertas situaciones en las que individuos pertenecientes a diferentes grupos sociales se ven involucrados, en particular cuando un blanco de clase alta hace evidente su racismo contra 


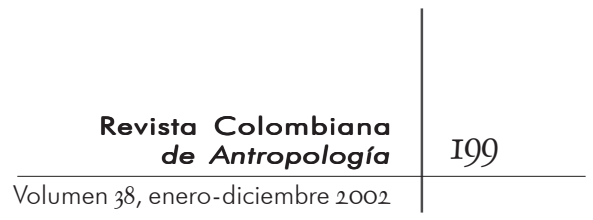

los negros y los indígenas, o su desprecio contra los mestizos pobres y los llama indios o negros. Aquí, los mecanismos de exclusión social y discriminación basados en estereotipos de clase, color, raza y etnia se entrelazan para cumplir un papel central en la definición de jerarquías.

Esto sucede porque el mestizo no puede ser aprehendido en términos de etnicidad, sino en términos raciales, culturales y de color. En este sentido, el color articula raza y etnicidad, siempre dentro de una estructura social, económica, política y cultural determinada -local, regional, nacional e, incluso, internacional-. Por tanto, la categoría de color posee un estatus particular en el análisis de la dinámica social de las sociedades latinoamericanas, similar al que poseen las categorías de raza y etnicidad. Un estatus, por cierto, que sólo puede ser comprendido -en la doble acepción del término- en el contexto de la estructura de clase social y económica de estas sociedades, puesto que es indudable que en los países latinoamericanos la valoración social del color en la escala blanco-negro se corresponde, si bien no siempre de modo unívoco, con el poder económico y el estatus social de los diferentes grupos sociales, así como con su proximidad con lo que se percibe como europeo y parte de la cultura occidental.

Así, mientras que las reglas que regulan el uso de los términos de color median la reproducción de un sistema social de discriminación y exclusión/inclusión, el blanqueamiento cultural y biológico se considera un medio de escape de la pobreza y la ignorancia, para escalar en la jerarquía social, ganar acceso al poder económico y político y lograr un mayor estatus social y cultural. En este sentido debe entenderse a Lugo (I995) cuando señala que en Latinoamérica el énfasis sobre el color como una categoría específica diferente de las de raza y etnicidad es complementario, mas que contradictorio, a los análisis sociales basados en clase, raza y etnia. La categoría de color, al igual que las de raza y etnicidad, es una categoría analítica por medio de la cual pueden analizarse y revelarse los procesos y problemas sociales. Es también un término que estructura y es estructurado por el orden social jerárquico de las sociedades latinoamericas. Sin embargo, mientras que las jerarquías de color han sido impuestas y rechazadas en toda Latinoamérica desde tiempos coloniales, la articulación de color como una categoría analítica en sus propios términos, esencial como las de raza y etnicidad para el entendimiento crítico de estos procesos, está todavía 
por hacerse. Los trabajos de Lugo y de Lancaster apuntan en este sentido. Dicho de otro modo, el color, tal como se ha intentado determinar aquí, tiene una doble existencia, como categoría analítica y como categoría práctica, cuyo potencial explicativo de procesos sociales y culturales en contextos latinoamericanos coloniales y poscoloniales no ha sido objeto de reflexión teórica suficiente.

\section{UNA APROXIMACIÓN AL SISTEMA SOCIAL DE LA AMAZONIA OCCIDENTAL}

OS ESTUDIOS HISTÓRICOS SOBRE LA AMAZONIA COLOMBIANA MUEStran la conformación de una sociedad regional periférica -durante cuatro siglos- con su perfil y ritmo propios, en el que deben insertarse las mismas sociedades indígenas (Llanos y Pineda, 1982; Llanos, 1987). En este sentido, la historiografía amazónica contemporánea presenta un descentramiento de las historias nacionales, aun cuando forma parte de ellas. El análisis de las construcciones del significado del color y de las relaciones de poder que mantienen o revierten las jerarquías del mismo en esta región de colonización hace necesaria la consideración, aunque sea somera, de los contextos históricos específicos y de las estructuras sociales e institucionales que lo enuncian.

La preocupación sobre la especificidad histórica de la enunciación del color en esta región plantea la necesidad de una periodización, dada la amplitud y la complejidad de esta historia, lo cual nos remite al problema de la determinación de intervalos que permitan reconstruir las relaciones y las dinámicas sociales y políticas en las que el color emerge y se transforma para señalar la posición de los sujetos. En lo que aquí concierne, ambas, la intensidad del mestizaje físico y cultural y las diferentes significaciones que se dan históricamente al blanco como agente y color primordial en el espacio de la construcción de las relaciones étnicas y raciales en la región de estudio, serán los criterios por tener en cuenta. 


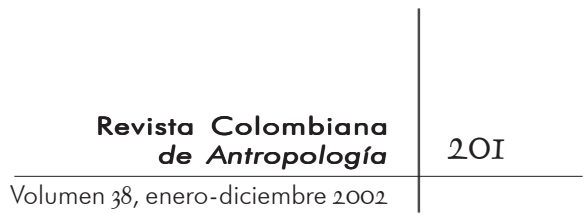

\section{Misioneros y caucheros}

S I SE TOMA COMO CRITERIO LA INTENSIDAD DE LA INTERACCIÓN INTERÉT$\mathcal{S}$ nica y del mestizaje, la historia regional puede dividirse, grosso modo, en dos grandes periodos. El primero corresponde al dominio colonial misional, que abarca casi cuatro siglos -dieciséis a diecinueve-. La empresa misional colonial, iniciada por los franciscanos en toda la amazonia en el siglo dieciséis, se derrumbó a mediados del diecinueve debido a la férrea resistencia indígena y a las luchas civiles en que se trenzaron los grupos políticos de la naciente república. Ello significó el abandono de estos territorios por parte de los misioneros franciscanos, con lo cual los habitantes indígenas de la región quedaban libres del dominio del blanco al tiempo que se reducía de manera importante la intensidad del contacto entre los dos.

Posteriormente, hacia finales del siglo diecinueve y comienzos del veinte, la región amazónica adquirió significado económico de nuevo, con la explotación de quina y caucho para el mercado mundial. La presencia de los blancos aumentó a medida que los recolectores de estos productos entraron en el área y los comerciantes de caucho establecieron el sistema del endeude, en el que cada recolector reclamaba un territorio y forzaba a los indios a volverse recolectores mediante un sistema de peonaje y esclavización sustentado en una espiral de deudas por mercancías adelantadas (Pineda, I980; Taussig, I987). El segundo periodo, que aquí llamamos moderno, empieza con la bonanza del caucho de comienzos del siglo veinte y se prolonga hasta hoy.

A grandes rasgos podemos decir que el periodo colonial misional se caracteriza por la ausencia casi total, o la existencia marginal, de procesos de mestizaje -biológico- en la interacción interétnica, de manera que puede hablarse, de manera general, del indio y del blanco como los actores centrales. El periodo moderno, en contraste, se caracteriza por una intensidad marcada y creciente de estos dos procesos, es decir, de mestizaje biológico e interacción interétnica. En este último periodo el colono irrumpe en el escenario, por así decirlo, y crea una nueva forma de relaciones que implica un proceso de interacción interétnica y de mestizaje, cada vez más dinámicos.

La misma dinámica del proceso social y de identidad-identificación que lo acompaña hace necesaria la división de este 
segundo periodo en dos subperiodos, el primero de menor intensidad de interacción interétnica que el segundo. El primero está determinado por la explotación del caucho y el control misional capuchino, y va desde comienzos del siglo veinte hasta bien entrados los años I950, cuando empezó la colonización campesina; el segundo comenzó con esta última y llega hasta el presente con las migraciones más recientes de miles de cultivadores y raspadores de hoja de coca. Hablamos del primer subperiodo del periodo moderno como el del caucho y la misión capuchina, y del segundo como el de la colonización y la coca, respectivamente.

Ahora bien, el hecho de que en este segundo periodo, que llega hasta hoy, no sólo se intensifique la interacción y el mestizaje entre indígenas y colonos, sino que, además, se instauren de manera simultánea y definitiva los discursos de la nación y el mercado propios de la modernidad en la amazonia, obligan a hacer un recuento más detallado de la dinámica de las relaciones sociales y los cambios sociopolíticos que lo determinan y lo caracterizan. Ello por cuanto es sólo con estos cambios y esta dinámica como telón de fondo que resulta pertinente un análisis de las relaciones interétnicas, las identificaciones culturales y las representaciones sociales de indígenas y colonos desde la perspectiva que aquí interesa. Esta última reflexión se hace más adelante.

\section{Colonización campesina y economía de la coca}

S ÓlO DESDE lOS AÑOS I95O, CUANDO INNUMERABLES FAMILIAS Y CAM$\checkmark$ pesinos se vieron obligados a abandonar sus regiones de origen y a buscar refugio en el piedemonte amazónico -como consecuencia de la conjunción de la crisis del minifundio en algunos departamentos vecinos y del recrudecimiento de la violencia política en otros- comenzó una verdadera colonización agraria en la región. En Putumayo, este proceso de expansión de la frontera agrícola contó, además, con un factor adicional que impartió mayor dinámica al proceso mismo de migración: el inicio de la explotación de petróleo, que atrajo hacia la región a una población de diverso origen, la cual permaneció en esta, ligada a la colonización agrícola una vez terminado el auge de demanda de 


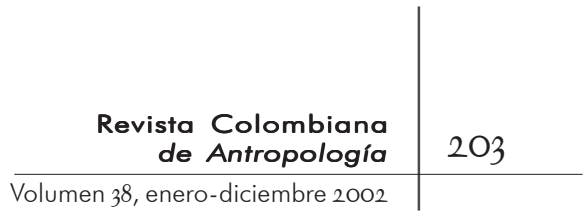

mano de obra que implicaba la instalación de la planta industrial de la explotación petrolera. Ha sido pues por la vía de la colonización agrícola que miles de campesinos desarraigados han llegado y continúan llegando al piedemonte amazónico de manera ininterrumpida, en busca de tierra y medios para establecerse. No está de más anotar que las instituciones del estado siempre han venido a la zaga del proceso de colonización.

Los años 1960 y 1970, posteriores a este primer momento, se caracterizan por el afianzamiento de estas colonizaciones y por el apoyo marginal del estado a las mismas en todo el piedemonte oriental, como una estrategia que busca eludir la puesta en marcha de una reforma agraria verdadera. Este periodo se caracterizó también por una legislación político-administrativa y de tierras que implicaba la discriminación económica, social y cultural de los indígenas frente al campesino colono y de cara al estado. A pesar de la precariedad de la presencia institucional estatal, lo cierto es que el referente del campesino-colono que trabajaba para el desarrollo ocupó un espacio preponderante en el imaginario que por ese entonces construía la región dentro de la nación. En cambio, la población indígena soportaba la discriminación social y cultural que imponía la vigencia de la ley 89 de I890, rectora de sus relaciones con el estado. Dicha ley ubicaba a la población indígena en una relación tutelar que muchos indígenas intentaban esquivar situándose como productores campesinos y mestizándose con los forasteros, si no física al menos culturalmente. Así, las relaciones entre indígenas y colonos se desenvolvieron al vaivén de las dinámicas cotidianas que los aproximaban, como en las tomas de yagé (Taussig, I987) o en los matrimonios, cada vez más frecuentes, y las diferencias de estatus, que en el caso de los colonos se asociaba a la condición de blanco, que los distanciaba. El campesino colono de este periodo se percibe y es denominado como blanco, sin importar a veces su bajo estatus social y económico, y como tal se halla en mejor posición que cualquier indio.

En la década de 1980, a medida que el estado intentaba ejercer un mayor control sobre la región, dos fuerzas inusitadas competían con él por la hegemonía en la zona. De una parte, la economía de la coca, que articuló a toda la sociedad regional y expandió las relaciones de producción capitalista a lo largo y ancho de la región, y que en las tres décadas recientes ha logrado generar una cultura regional, rural y urbana, que atraviesa las 
diferencias culturales que por un tiempo marcaron las relaciones pero también las diferencias entre indígenas y campesinos colonos. En efecto, la complejidad de las nuevas relaciones sociales en el marco de la compresión temporo-espacial que traen consigo la globalización y articulación de la región con los mercados nacionales e internacionales, imparte una nueva dinámica a las relaciones interétnicas en la región, de modo que indígenas y campesinos adquieren un mismo estatus económico y social -en tanto productores de base de coca- (Chaves, I996). De otro lado, la expansión de esta economía ilegal proporciona el soporte económico a los grupos armados al margen de la ley, que en su competencia por el control territorial y de la población local ponen en marcha procesos de ordenamiento de amplias áreas de la región, que permiten su consolidación en la misma al tiempo que recrean las condiciones de la economía ilegal que posibilita su financiación. En este contexto la población local se debate entre buscar los favores del estado, cada vez más controlado por una elite regional emergente, y evitar el control de la guerrilla y los paramilitares (Ramírez, 200I).

Hay, además, un tercer momento propio de los años I980 y I990, que habría de plasmarse en el contenido de la constitución de I99I y en diversas leyes promulgadas a lo largo de la última década, que reglamentan, entre otros, los derechos territoriales, políticos y económicos de los grupos étnicos, pero que también llevan consigo los elementos ideológicos del imaginario que supone la construcción cultural y política de la nación inclusiva. De otra parte, es indudable que la generación de discursos indigenistas y ambientalistas en círculos técnicos, políticos, académicos e institucionales en las últimas décadas, y su apropiación por parte de las comunidades y los dirigentes indígenas en el contexto de su lucha por derechos culturales y territoriales, marca de manera particularmente profunda la dinámica social, económica, política y cultural de la región hasta los comienzos mismos del siglo veintiuno. La recuperación de estos momentos parece esencial para una antropología del color; lo que sigue es un intento en esa dirección. 


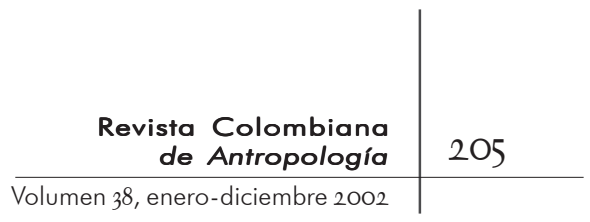

\title{
INTERACCIÓN INTERÉTNICA Y DISCURSOS HEGEMÓNICOS
}

L DISCURSO QUE SOPORTA LAS RELACIONES DE DOMINACIÓN DE LOS - colonizadores españoles, misioneros y comerciantes, es el de la civilización por medio de la cristianización o de la utilización de su fuerza de trabajo. En el marco de este proyecto civilizador se construyen las clasificaciones de blancos e indios del primer periodo.

Taussig, en su discusión del significado del término blanco en el contexto del periodo de la cauchería y la misión capuchina, observa que de acuerdo con Joaquín Rocha, un viajero colombiano de comienzos del siglo veinte,

\begin{abstract}
los habitantes del Putumayo estaban divididos en dos grandes clases o tipos sociales: los blancos y los 'indios salvajes'. La categoría de blancos (también referida como 'racionales', cristianos y 'civilizados') incluía no sólo gente fenotípicamente blanca, sino mestizos, negros, mulatos, zambos, e indios incorporados a la civilización desde los tiempos de la conquista española (Taussig, I992: 155).
\end{abstract}

Para precisar lo anterior el autor trae a colación a Simson, un viajero inglés de la época, para quien "los puros indios" de las selvas estaban divididos en indios e infieles. "Los indios hablan quechua, consumen sal, y son semi-cristianos, mientras que los infieles, también conocidos como "aucas", hablan otras lenguas diferentes, rara vez comen sal y no conocen nada acerca del bautizo de la iglesia católica” (Taussig, I992: I55). Aquí, entonces, la categoría de blanco se aplicaba a un conjunto heterogéneo de individuos que comparten la característica de haber sido civilizados en algún grado por los colonizadores europeos. La diferenciación entre indios e infieles señala la importancia que había asumido la cristiandad en la cultura de la conquista. "La distinción entre indio cristiano y pagano se volvió ideológicamente decisiva debido a su importancia para facilitar los legalismos de la esclavización y el uso de la fuerza militar" (Taussig, 2002: I86).

A diferencia de lo ocurrido en Brasil y Perú, donde una vez terminado el auge de la explotación cauchera se dio el caso de remanentes importantes de población foránea que permanecieron en el área interactuando con las poblaciones indígenas y 
generando un mestizaje biológico y cultural auténtico -los $\mathrm{Ca}$ boclos (Nugent, I993) y ribereños (Gow, I99I)-, en Colombia la mayoría de los colonizadores de ese periodo abandonó la región. Esto explica en parte por qué, en el caso colombiano, no surgió un mestizo amazonense, al tiempo que ofrece una explicación de por qué la categoría de colono blanco continuó utilizándose en la amazonia colombiana para denominar a todo aquel que hubiera migrado hacia la región, sin importar su tiempo de permanencia ni su proximidad física y social con los indígenas autóctonos, como tampoco su procedencia étnica.

La disputa por el dominio que impusieron los caucheros peruanos por medio de la Casa Arana en partes de la amazonia colombiana culminó con el conflicto entre los dos países en I932. Fue entonces cuando el gobierno colombiano mostró algún interés por la región, lo cual vino acompañado de la presencia marginal y no siempre permanente de algunas instituciones civiles y militares del estado. Apoyado en los misioneros católicos capuchinos a los que había encomendado el control de esos territorios desde los inicios del siglo, promovió la construcción de caminos y carreteras por los que años más tarde habrían de penetrar las corrientes migratorias de campesinos provenientes de otras regiones del país.

Fueron pues los misioneros católicos -capuchinos en Caquetá y Putumayo y montfortianos en Guaviare-, y no el estado, los encargados de inculcar entre los pobladores la colombianidad, el sentimiento nacionalista, con el propósito de competir con Perú y Brasil en la colonización de esos territorios fronterizos. La institución principal de internados misionales, la imposición/ enseñanza del español, la cristianización y la introducción de pautas de la economía monetaria fueron los medios principales utilizados por los misioneros capuchinos para lograr el control férreo de la población indígena y la articulación efectiva de las poblaciones autóctonas con las dinámicas regionales que se abrían al mercado. Igualmente importante resultó su intervención en la producción de una imaginería sobre los indios, en la que convergía el primitivismo de sus 'costumbres salvajes' con sus 'poderes sobrenaturales' para la curación mediante la brujería, y que con el correr del siglo aproximaría a colonos blancos e indios en las 'tomas' de yagé, el espacio privilegiado de la representación de la alteridad en esas regiones. Como resultado de este doble proceso, hacia finales de este periodo tenemos 


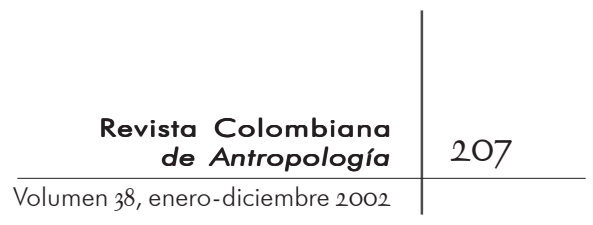

que si bien es cierto que las culturas de los indígenas y los colonos blancos se presentan como manifestaciones claramente diferenciadas, también la aproximación física y cultural de ambos grupos en los centros de mayor contacto y su entrecruzamiento son un hecho.

En el proceso, las categorías sociales que organizan y ubican los diferentes grupos de actores en la jerarquía regional, su significado y su relación mutua, se han ido transformando para este periodo. Así, mientras que la categoría de blanco siguió utilizándose de manera general para denominar a todo aquel considerado portador de una cultura occidentalizada o civilizada, como se la denominaba corrientemente, y que desde la perspectiva de los indios significaba, además, de fuera de la región, no ocurrió lo mismo con la categoría de indio. De hecho, esta última llegó a abarcar un espectro más amplio de nativos que aquellos a quienes Simons denominaba infieles. Para decirlo de otra manera, en el proceso de mestizaje físico y cultural la categoría de infieles/auca tiende a desaparecer o a ser parte sólo del imaginario que permite a los indios representarse a sí mismos como civilizados frente a los blancos, con el fin de no ser discriminados. En contraste, la categoría de indio comprende ahora a todo aquel que hace parte de una comunidad con costumbres y lengua propia, pero también a los indios que viviendo por fuera de sus comunidades reconocieran un ancestro en una de ellas.

\section{RELACIONES INTERÉTNICAS \\ Y REPRESENTACIONES SOCIALES}

D ESDE LA PERSPECTIVA QUE AQUÍ INTERESA, LA PRESENCIA DEL COLONO en el periodo que comienza con la bonanza del caucho sugiere un problema que ya se daba en el periodo histórico anterior, pero que se hace más complejo ahora. Se trata de la pregunta que surge al respecto de quién es (el) indio, y quién es (el) blanco, y que se plantea ahora, además, en el periodo de la colonización y la coca con respecto a quién es (el) colono. Ocurre entonces que la calificación de sujeto étnico no acompaña exclusivamente a las etnias propias del territorio, puesto que en algunos casos también debe utilizarse para referirse tanto a indígenas migrantes/colonos -emberás-chamí, paeces, tukanos, entre otros- como 
a indígenas que hacen parte de uniones matrimoniales con no indígenas, y que por consiguiente deberían considerarse, en este caso, mestizos desde un punto de vista racial y cultural. De igual manera, la denominación de colono tampoco se refiere únicamente a los migrantes de fuera, pues lo que se conoce como el ciclo del colono determina para algunos de estos su condición de migrantes dentro de la frontera de colonización. En este último caso es probable que la denominación de blanco no sirva tampoco para referirse al colono marcado por la diferencia étnica (no mestizo). Esto sólo para señalar algunas de las dificultades para procesar los límites de una u otra categoría.

A manera de hipótesis, entonces, es posible pensar que durante el periodo del dominio misional colonial la dinámica de la relación de dominación se enmarcaba de manera general dentro de los referentes étnicos y culturales, y si se quiere de raza y color, que construyeron conquistadores e indios para identificarse y representarse mutuamente, y que estos referentes fueron relativamente estables en el tiempo -el blanco, para el colonizador, y el no blanco o indio, para el colonizado-. Esta interdependencia entre el blanco y el indio es jerárquica, en la que el primero es el término dominante que subordina al segundo; uno y otro construyen sus propias representaciones de sí mismo y de su otro, pero esa construcción se da en una relación de poder que el indio del periodo de la misión colonial no logra desestabilizar. Esta es la razón por la cual la categoría blanco tiene sentido en la formación discursiva del mestizaje, pero principalmente como el momento determinante del blanqueamiento, en el que la blancura se naturaliza, crea su verdad y legitima la autoridad.

En contraste, para el periodo de la colonización y la coca, que es el que nos ocupa de manera particular, las cosas parecen no ser tan claras, por cuanto los criterios que sirven de referente para fijar las divisiones de exclusión-inclusión se hacen borrosos y confusos. Me explico. En el periodo del caucho y la misión capuchina hay al parecer un discurso del blanco inmigrante, quien por su posición de poder político y económico en la relación con los otros actores de la región se sitúa por fuera de y encima del colono (campesino-extractivista) y del indio originario de la amazonia. De manera simultánea, parece haber un discurso del indio originario de la región, quien ve al blanco y al colono como inmigrantes y a quienes denomina de manera ge- 


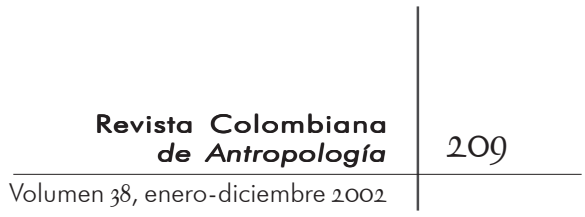

neral como blancos. El colono, pues, pertenece o no al mundo del blanco según la forma en que se articule la perspectiva específica desde la cual se lo mire. En general, puede pensarse que el colono se sitúa a sí mismo como blanco frente al indio, al tiempo que se sabe excluido del mundo del blanco dominador.

Hacia el final de este periodo, el cual corresponde a los desarrollos más recientes en la región, se ha llegado entonces a una resignificación de los discursos identitarios de indígenas y colonos como resultado de la mayor intensidad de las relaciones interétnicas y de la dinámica socioeconómica. En general, el blanco conserva y refuerza su posición de poder político y económico por encima de los actores subalternos en la región, el colono (campesino) y el indígena. Por su parte, estos últimos se encuentran en una posición de desventaja frente al blanco, en la medida en que conviven en condiciones socioeconómicas similares más o menos precarias, en las que la intensidad del mestizaje y la interacción cotidiana es cada vez mayor. Esta dinámica social, relativamente reciente, crea las condiciones para la construcción de un nuevo discurso más diferenciado por parte del colono y del indio, en el que el indio reconoce la exclusión del colono del mundo del blanco -dominador-, algo que no era evidente en el periodo del caucho y las misiones capuchinas, y ambos se reconocen a sí mismos y mutuamente en su posición subalterna frente al blanco. La cercanía del colono y el indio permiten, ahora, que el colono haga parte de una comunidad indígena sin que ello implique que sea aprehendido como un individuo étnico, al menos no de un modo genuino -el caso de Jaime Erazo que mencionaba en la Introducción-. En este sentido es que los referentes de exclusión-inclusión se hacen aquí borrosos y difusos y, por qué no, confusos; pero que al mismo tiempo se constituyen en el elemento esencial para la estructuración de un sistema regional de clases.

En un periodo más reciente, la constitución trastocó en cierto modo la lógica que subyace a los procesos de mestizaje y blanqueamiento esbozada arriba, en la medida en que imparte una nueva dinámica a la construcción de imaginarios étnicos -la re-creación de viejos (memoria) y nuevos (invención) referentes de inclusión/exclusión étnica- entre grupos e individuos subalternos, sin que los discursos que estos últimos puedan haber elaborado en este nuevo contexto hayan llegado a desestabilizar la relación de poder y representación sobre la que se 
construye el sistema de clases regional y dentro de la cual ocurren el mestizaje y el blanqueamiento.

La pregunta que se plantea es, ¿cómo opera esta doble lógica de inclusión-exclusión, de de-marcación social -en el sentido de que marca y demarca espacios sociales- propia del periodo histórico de la colonización y la coca? Es indudable que la relación de poder en la que se enmarcan los referentes culturales, étnicos, raciales y de color adquiere aquí importancia crucial en la construcción positiva o negativa de la elite blanca y, así mismo, de las clases subalternas de indios y colonos, como subalternos de color.

\section{ESQUEMA PARA UNA TIPOLOGÍA DE LOS INDÍGENAS Y COLONOS}

EN LA AMAZONIA OCCIDENTAL COLOMBIANA

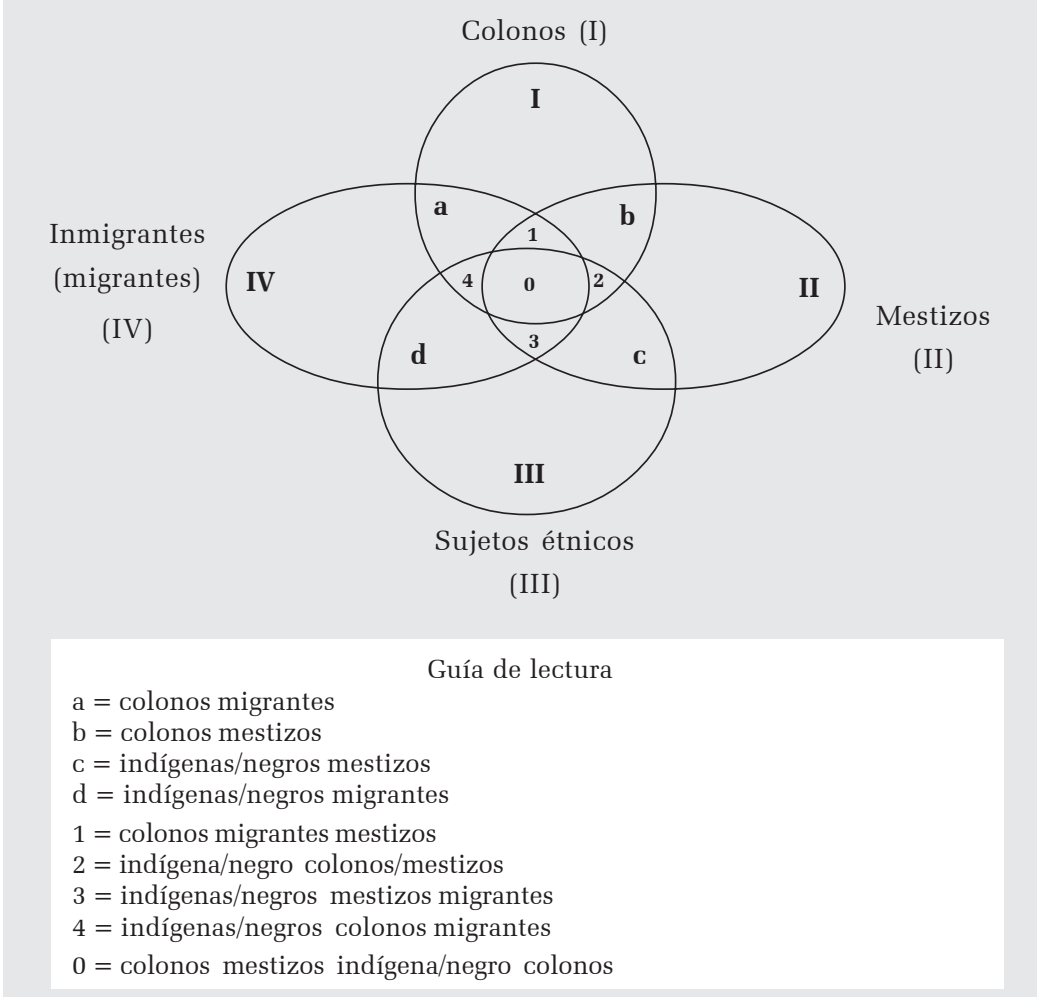

La denominación colonos migrantes hace referencia a aquellos sujetos que no han consolidado su colonización y continúan migrando dentro (o hacia fuera) de la región. 


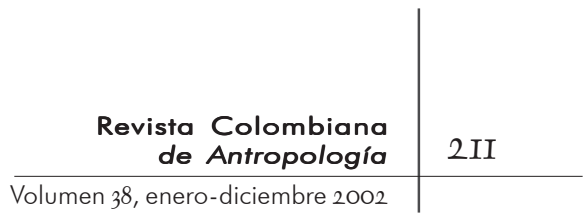

Ahora bien, el proceso del mestizaje plantea en este contexto un nuevo problema que no puede ser resuelto completamente. La presencia del inmigrante, del colono campesino, del indio étnico y del mestizaje que caracterizan la dinámica social de la región implican una sobreposición o traslapamiento de (estos) diferentes 'atributos' permanentes-temporales o contextualesposicionales de los distintos actores, como momentos determinantes en la creación dialógica (relacional) de la identidad. En este sentido debe leerse el esquema siguiente, como un intento de visualizar la relación de los elementos que estructuran esa creación dialógica.

La dificultad del análisis de las jerarquías dentro de un mismo grupo surge, entonces, de que dichos atributos y la posición económica o política se entrecruzan en contextos sociales diversos; y es justamente el color en tanto categoría analítica y práctica la que resuelve esa dificultad. El color, la particularidad de las apariencias físicas, asociadas a características raciales y a la posición social de los sujetos, cumple un papel central en estos contextos sociales en los que tiene lugar la estructuración de relaciones de discriminación, justamente porque media y articula los distintos atributos de inmigrante, colono, mestizaje y etnicidad, que por sí mismos no son suficientes para crear y fijar una determinada estructura social jerarquizada: la matriz social del mestizaje.

Sólo así es posible entender los procesos de discriminación social que se presentan desde hace una década en el marco de los procesos de re-indigenización de campesinos colonos en diferentes partes de la amazonia, en los cuales el papel del estado ha sido central. El aumento de las cifras de población y de cabildos indígenas en los censos poblacionales recientes ha generado desconcierto entre las autoridades estatales nacionales y regionales, y entre los miembros de las etnias indígenas autóctonas que hoy compiten por los recursos del estado (véase Chaves, 2003).

Para el análisis que aquí interesa, cabe resaltar que el proceso descrito muestra el modo particular en que comunidades aparentemente integradas dentro de las dinámicas campesinas mestizas regionales recurren a construcciones identitarias como el medio que posibilita su inclusión en el espacio de aparente reconocimiento que la constitución de I99I abrió para las comunidades indígenas. Sin embargo, al tiempo que estas articulan sus nuevas perspectivas identitarias, el estado, en un intento por 
frenar y controlar ese proceso de inclusión, ha establecido criterios técnico-políticos para señalar las características de los que, a su manera de ver, constituyen los verdaderos sujetos étnicos, dignos de un tratamiento 'preferencial' de parte suya. Las tensiones generadas a partir de aquí conducen a la emergencia de diversos modos, de diversas categorías de indígenas, en las que el elemento central de la definición lo constituye la presencia de la lengua, que se constituye en el criterio de mayor estatus identificatorio de los indígenas. Según esto, es posible identificar entonces: indígenas autóctonos (véase el diagrama, III), que hablan su lengua, en razón de lo cual su diferencia cultural resulta incuestionable; indígenas autóctonos que ya no hablan la lengua pero que encuentran espacios de alianza política con los anteriores (c); indígenas migrantes que hablan la lengua (d) e indígenas migrantes que no la hablan, pero que todavía lo es en sus regiones de origen (3), lo que hace que su identidad no sea puesta en entredicho; e indígenas que no hablan la lengua y que no disponen de los referentes que permiten a los otros indígenas construir su identidad étnica, para construir la suya propia (4).

Dentro de este último grupo la permanencia del color se evidencia como el referente sobre el que se construye un discurso que permite identificarse como indio, señalar su ascendencia indígena, los rasgos físicos asociados con la misma y, por supuesto, su posición subordinada. Dicho de otro modo, es justamente cuando se hace manifiesto el poder discriminatorio de la ausencia de los atributos del reconocimiento étnico que su identidad comienza a ser enunciada categórica y prácticamente mediante el color.

-María Inés, ¿cómo supo usted que pertenecía a la etnia pasto?

-Pues resulta que en una reunión que tuvimos en el cabildo que se disolvió comenzamos a mirarnos y a ver que todos éramos como parecidos; mire la cara de este se parece a este, los ojos, los cachetes, la piel, el pelo, y todos nos parecíamos. Y luego yo pregunté, a ver, cuáles son sus apellidos y entonces iban apareciendo los Cueltán, Chapuel, Tutalcha Tenganan, Cuarán (como yo), todos de Nariño, y yo me di a la búsqueda de qué podíamos ser, y ahí fue que supe que éramos pastos (entrevista a María Inés Cuarán, gobernadora del cabildo Siberia del municipio de Orito). 


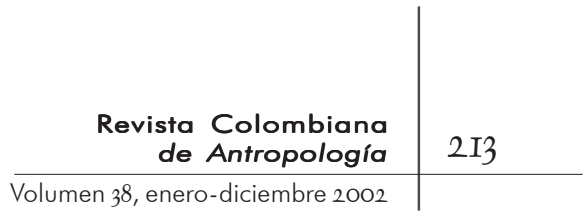

-¿Usted, cuando era joven se identificaba como indígena?

-A mí, yo nunca me he sentido que sea blanco, yo nunca. Porque desde mi tierra, como le digo, nos llamaban naturales era porque éramos indios, y yo nunca me sentí ni blanco ni mestizo. Y de acuerdo a las tradiciones, si a mí me ponen a rellenar un papel, yo lo hago con lo que me contaba mi papá (entrevista con Félix Canacuán, cabildo Huasipungo de Villagarzón)

Es decir, dentro de los márgenes ambiguos que crea el mestizaje biológico y cultural los procesos de reindigenización o reetnización que hoy están a la orden del día en todo el país reviven los privilegios y posibilidades de los de piel blanca, pero también la subordinación y la marginación de la gente no blanca, india o de color.

\section{REFLEXIONES FinALES}

N BUEN NÚMERO DE ANTROPÓLOGOS QUE TRABAJA SOBRE LA AMAZOnia utiliza los términos mestizo, indio y blanco como si fueran categorías sociológicas autoevidentes, en un esquema que puede resumirse como sigue: indios son aquellas personas que son indígenas (autóctonos) del área, portadores de culturas auténticas hasta que las fuerzas de la aculturación barren con ellos. Los blancos, o la gente blanca, son los inmigrantes, europeos o criollos, dominadores y explotadores, portadores de una cultura ajena al área. Los mestizos son una categoría problemática, una mezcla de inmigrantes pobres y de indios deculturados que olvidaron su lengua y, por tanto, no pueden ser portadores de culturas indígenas auténticas.

La literatura sobre las clasificaciones culturales de las gentes de la amazonia occidental, en gran parte preocupada por la autenticidad de comunidades particulares, ha ignorado este hecho. La gente de la amazonia utiliza continuamente términos como indio y colono, y referencias al color como blanco o no blanco como parte de una práctica social en proceso; por lo mismo, no significa ni es lo mismo ser blanco hoy que hace unas décadas en Guaviare, que en Caquetá o Putumayo. Estos términos se usan no para definir gente en términos culturales abstractos sino para ubicarlos en unas relaciones sociales específicas, siempre cambiantes tanto en relación con aquellos 
que los utilizan como con los que esos términos designan. Los términos indio, colono, blanco y no blanco se utilizan para ubicar la gente local dentro de posiciones particulares en la jerarquía del poder socioeconómico en la región.

Tal como dicen Gow (I99I) y Taussig (I987), categorías étnicas como blanco e indio no son sólo términos que refieren diferentes tipos raciales, sino que son construidas y pertenecen a sistemas específicos de creencias y representación. En este sentido, son categorías étnicas y sociales inestables. Los dos autores señalan, además, que tales categorías se construyen de manera diferente en cada contexto histórico específico. Ambos arguyen que el término blanco, en el sistema de clases y castas de la amazonia, sólo puede ser entendido en relación con el término indio, y del mismo modo, el término mestizo en relación con los dos anteriores. Puesto así, la pregunta que se plantea hoy para el contexto amazónico colombiano es la siguiente: si los colonos que llegaron al piedemonte amazónico en los años I950, al inicio del periodo que hemos llamado de la colonización y de la coca, se identifican hoy por hoy como indígenas, en un proceso que conjuga la recuperación de la memoria histórica con procesos políticos acompañado de un proceso más sutil de refinamiento de esta auto-percepción que los lleva gradualmente a identificarse como pertenecientes a una u otra etnia, ¿a quienes se refieren estos, indios, colonos, cuando utilizan la categoría blanco?

\section{BIBLIOGRAFÍA}

BARTH, FREDRIK. I986. "Introducción”. Los grupos étnicos y sus fronteras. Fondo de Cultura Económica. México.

Chaves, Margarita. i998. "Identidad y representación entre indígenas y colonos de la amazonia colombiana”. En María Lucía Sotomayor (editora). Modernidad identidad y desarrollo. Ican-Colciencias. Bogotá.

- 200I. "Discursos subalternos de identidad y movimiento indígena en el Putumayo". En Mauricio Archila y Mauricio Pardo (editores). Movimiento sociales, Estado y democracia en Colombia. CES/Universidad Nacional de Colombia-ICANH. Bogotá.

- 2003. "Cabildos multiétnicos e identidades depuradas". 


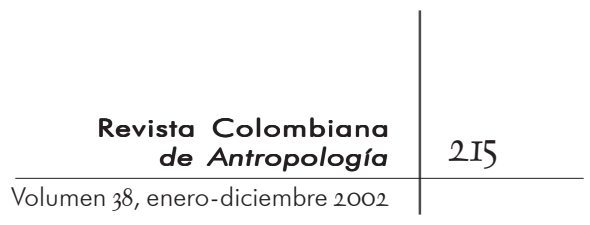

En Fronteras, metáforas y territorios. INER-Universidad de Antioquia. Medellín. En prensa.

Gow, Peter. I99I. Of mixed blood. Kinship and history in Peruvian Amazonia. Clarendon. Oxford.

Hall, Stuart. I990. "Cultural identity and diaspora”. En J. Rutherford (editora). Identity, community, culture, difference. Lawrence \& Wishart Limited. Londres.

. I99I. "Old and new identities, old and new ethnicities". En Anthony King (editor). Culture, globalization and the worldsystem. State University of New York. Binghamton.

KLOR DE Alva, Jorge. I995. "The postcolonization of the (Latin) American experience. A reconsideration of 'colonialism', 'postcolonialism', and 'mestizaje’”. En G. Prakash (editor). After colonialism: imperial histories and postcolonial displacements. Princeton University Press. Princeton.

LANCASTER, Roger. I992. Life is hard. Machismo, danger and the intimacy of power in Nicaragua. University of California. Berkeley.

Llanos, Héctor. I987. “Tiempos y espacios coloniales amazónicos”. En Colombia amazónica. Universidad Nacional de ColombiaFondo FEN. Bogotá-

Llanos, Héctor y Roberto Pineda C. I982. Etnohistoria del Gran Caquetá. Siglos (XVI-XIX). Banco de La República, Fundación de Investigaciones Arqueológicas. Bogotá.

Lugo, Alejandro. 1993. "Fragmented lives, assembled goods: a study in maquilas, culture, and history at the Mexican borderlands”. Disertación no publicada. Department of Anthropology. Stanford University.

Nugent, Stephen. 1993. Amazonian Caboclo Society. An essay on invisibility and peasant economy. Berg Publishers. Oxford.

Pineda, Roberto. I980. Historia oral y proceso esclavista en el Caquetá. Finarco, Banco de la República. Bogotá.

Ramírez, María Clemencia. 200I. Entre el Estado y la guerrilla: identidad y ciudadanía en el movimiento de los campesinos cocaleros del Putumayo. ICANH-Colciencias. Bogotá.

SAnSONE, Livio. I997. "The new blacks from Bahia: local and global in afro Bahia”. Identities. Volumen 3: 4.

Stutzman, Ronald. I98I. "El mestizaje: an all-inclusive ideology of exclusion”. En Norman Whitten (editor). Cultural transformation and ethnicity in Modern Ecuador. University of Illinois Press. Urbana. 
Taussig, Michael. 1992. "Culture of terror"Space of death: Roger Casement's Putumayo Report and the explanation of torture". En Nicholas Dirks (editor). Colonialism and culture. The University of Michigan Press. Ann Arbor.

- 2002 (1987). Chamanismo, colonialismo y el hombre salvaje. Un estudio sobre el terror y la curación. Traducción de Hernando Valencia. Norma. Bogotá.

WADE, Peter. 1993. Blackness and race mixture. The dynamics of racial identity in Colombia. John Hopkins University Press. Baltimore.

- 2000 (1997). Raza y etnicidad en Latinoamérica. Traducción de María Teresa Jiménez. Abya-Yala. Quito.

Whitten, Norman. 1985. Sikuangaruna. The other side of development in Amazonian Ecuador. University of Illinois. Urbana. 